\title{
Inventory Control in Supply Chains: Alternative Approaches to a Two-stage Lot-sizing Problem
}

\author{
András Kovács ${ }^{1}$, Péter Egri ${ }^{1}$, Tamás Kis ${ }^{1}$, József Váncza ${ }^{1,2}$ \\ ${ }^{1}$ Fraunhofer Project Center for Production Management and Informatics, \\ Computer and Automation Research Institute, Hungarian Academy of Sciences \\ 2 Department of Manufacturing Science and Technology, \\ Budapest University of Technology and Economics, Hungary \\ \{andras.kovacs,egri,tamas.kis,vancza\}@sztaki.hu
}

December 15, 2011

\begin{abstract}
The principal challenge of inventory control in supply chains is that the interacting autonomous enterprises have to plan their production and logistics under information asymmetry, driven by different, often conflicting objectives. In this paper, four different computational approaches are investigated to cope with this challenge: decomposition, integration, coordination, and bilevel programming. The four approaches are applied to solving the same two-stage economic lot-sizing problem, and compared in computational experiments. The prerequisites of the approaches are analyzed, and it is shown that the profits realized and the costs incurred at the different parties largely depend on the solution approach applied. This research also resulted in a novel coordination mechanism, as well as a new algorithm for the bilevel optimization approach to the investigated lot-sizing problem. A specific goal of this study is to highlight the so far less recognized application potential of the coordination and the bilevel optimization approaches for controlling inventories in a supply chain.
\end{abstract}

Keywords: Supply chain, inventory control, integration, coordination, bilevel programming.

\section{Introduction}

The principal challenge of inventory control in supply chains is that the autonomous enterprises have to plan their production and logistics under information asymmetry, driven by different, often conflicting objectives. Moreover, 
the individual enterprises typically make decisions that affect the entire supply chain, and for this purpose they also exploit private information that is inaccessible to the other parties.

This paper investigates four different approaches to cope with this challenge. According to the classical decomposition approach, each party optimizes its own production and logistic decisions without explicitly considering the consequences on the supply chain level. The integrated approach optimizes the overall performance of the supply chain by centralized planning, however, this requires a tight integration of the parties. By lifting the latter requirement, the coordinated approach seeks for mechanisms that motivate the autonomous enterprises to cooperate in finding mutually beneficial plans by negotiation and benefit sharing. Finally, the bilevel approach enables an individual party, in possession of sufficient information about its partners, to optimize its production taking into account the actions that it can expect from the partners.

The goal of this study is to provide a clear-cut comparison of the above fundamental approaches by applying them to a common problem model. The main modeling, computational, and managerial implications are investigated with a focus on the prerequisites of each approach, such as the availability of information, the contractual requirements, or the assumptions on the type of cooperation. Furthermore, the potential gains for the different parties of adopting a given approach are examined, and the resulting solutions, profits and costs are compared. To the best of our knowledge, this is the first study that provides a self-contained comparison of these approaches, applied to the same inventory control problem in different settings. A specific goal of the paper is to highlight the benefits of the two less recognized approaches, coordination and bilevel optimization, for the different parties in the supply chain. A new coordination mechanism (Section 5) and a new algorithm for solving the bilevel version of the investigated lot-sizing problem (Section 6) are also presented.

The investigated problem corresponds to an uncapacitated economic lotsizing problem in a two-echelon supply chain. In a dyadic situation where a buyer-supplier chain meets external demand, this problem involves both the production related decisions of the supplier, as well as the logistic decisions of the buyer. Although for the sake of analytical clarity some simplifying assumptions have to be taken, the basic problem has direct application relevance. Primarily, a retailer may assume the role of the buyer, connecting exogenous market demand and the service of the supplier. Further on, a similar buyer-supplier relationship may hold between multiple divisions of a large enterprise.

For a review of inventory control problems, both as faced by a single decision maker and in a supply chain, the reader is referred to [3]. The potential gain by integrated versus decentralized decision making in supply chains is investigated in [12], where the difference of the induced costs is defined as the price of anarchy. The coordination of supply chains consisting of autonomous enterprises is studied in detail in [2], while a comprehensive taxonomic survey of coordinated buyer-vendor models in a deterministic, time invariant setting is provided in [15]. The fundamental ideas of bilevel programming are presented in [7], and the application of this approach to the management of multi-divisional organizations 
is studied by Bard [4]. Further, more specific references are provided later in Sections 3 to 6 , each of which investigate one of the four possible computational approaches to the studied lot-sizing problem.

\section{Problem definition}

\subsection{A two-stage lot sizing problem}

The different computational approaches are studied on a two-stage single-item uncapacitated lot-sizing problem as follows. Let us consider a supply chain that provides a single item to its customers. The supply chain consists of two independent companies, a buyer and a supplier. The buyer (and hence, the supply chain) faces dynamic, deterministic external demand $d_{t}, t=1, \ldots, T$, over a discrete time horizon of $T$ time periods.

Departing from the known demand, the buyer computes its supply requests, i.e., the amount $x_{t}^{1}$ of the item that should be delivered from the supplier to the buyer in each time period $t$. The buyer may use the delivered amount partly to satisfy the demand in the same period $t$, partly to keep it on stock to cover future demand in periods $t^{\prime}>t$, and partly to satisfy backlogged demand from previous periods $t^{\prime \prime}<t$. Delivering a positive amount in period $t$ incurs a fixed cost of $f_{t}^{1}$ plus a per unit cost of $p_{t}^{1}$. Holding inventory and backlogging at the buyer take $h_{t}^{1}$ and $g_{t}^{1}$ per unit and per period cost, respectively. These delivery, holding, and backlogging costs are paid by the buyer to an external party.

The income of the buyer consists of the per unit purchase price $q_{t}^{1}$. Symmetrically, the buyer pays a per unit purchase price $q_{t}^{2}$ for the ordered goods. This purchase price is independent of the above logistic costs.

To cover the demand set by the buyer's supply requests, the supplier generates a production plan that specifies the amount $x_{t}^{2}$ of the item to be produced in period $t$ over the planning horizon. In each period $t$ where a positive amount $x_{t}^{2}>0$ is produced, production cost is incurred: a fixed setup cost of $f_{t}^{2}$ plus a per unit cost of $p_{t}^{2}$. Just as the buyer, the supplier can hold stock or backlog demand, for a cost of $h_{t}^{2}$ and $g_{t}^{2}$ per unit and per period, respectively. Moreover, it is assumed that the production and holding costs that occur at the supplier are paid by the supplier to an external party, whereas the backlogging cost is paid by the supplier to the buyer as a penalty for the delay caused.

Furthermore, it is assumed that all demand must be satisfied by the end of the horizon and no item remains in stock, i.e., $\sum_{t=1}^{T} d_{t}=\sum_{t=1}^{T} x_{t}^{1}=\sum_{t=1}^{T} x_{t}^{2}$. The production and delivery lead times are zero. The objective of both parties is to maximize their profits.

In all models studied in the sequel the decision variables of the buyer are the $x_{t}^{1}$ supply, $s_{t}^{1}$ inventory and $r_{t}^{1}$ backlog quantities for each time period $t=$ $1, \ldots, T$ of the planning horizon. The supplier has a decision problem of identical structure, with $x_{t}^{2}$ production, $s_{t}^{2}$ inventory and $r_{t}^{2}$ backlog quantities. Whenever appropriate, we distinguish the two parties with an upper index $k$, where $k=$ 1 stands for the buyer's and $k=2$ for the supplier's decision variables and 
parameters. Auxiliary binary variables $y_{t}^{1}$ and $y_{t}^{2}$ are introduced to capture events of delivery and production, respectively. The notation is summarized in Table 1.

\begin{tabular}{|l|l|}
\hline \multicolumn{2}{|l|}{ Dimensions } \\
\hline$T$ & Number of time periods \\
\hline Upper indices \\
\hline$\square^{1}$ & Parameters/variables related to the buyer (planned values) \\
$\square^{1 R}$ & Parameters/variables related to the buyer (realized values) \\
$\square^{2}$ & Parameters/variables related to the supplier (plans match realization) \\
\hline Parameters \\
\hline$d_{t}$ & External demand in period $t$ \\
$f_{t}^{k}$ & Fixed delivery $(k=1) /$ production $(k=2)$ cost in period $t$ \\
$p_{t}^{k}$ & Per unit delivery $(k=1) /$ production $(k=2)$ cost in period $t$ \\
$h_{t}^{k}$ & Per unit and per period holding cost at party $k$ in period $t$ \\
$g_{t}^{k}$ & Per unit and per period backlog cost at party $k$ in period $t$ \\
$q_{t}^{k}$ & Per unit purchase price at party $k$ in period $t$ \\
\hline Variables \\
\hline$x_{t}^{k}$ & Amount of goods requested by the buyer $(k=1) /$ produced by the supplier $(k=2)$ in \\
$y_{t}^{k}$ & period $t$ \\
$s_{t}^{k}$ & Binary variable indicating whether a positive amount is produced/delivered in period $t$ \\
$r_{t}^{k}$ & Backlog at party $k$ at the end of period $t$ \\
\hline Performance measures \\
\hline$C^{k}$ & Total production and logistic cost incurred at party $k$ \\
$P^{k}$ & Profit realized by party $k$ \\
\hline
\end{tabular}

Table 1: The notation used in the paper.

\subsection{Plans and realization}

Since the above model allows the supplier to backlog, according to some of the investigated approaches, the buyer may not be able to anticipate situations where the realized deliveries from the supplier deviate from the supply requests. Therefore, the executed scenario may differ from the plan, and the rules of the execution must be established. The following rules are applied.

If the supplier produces the goods on time, then the buyer must call off the amount indicated in the supply requests. Otherwise, i.e., if the supplier backlogs demand, then the buyer calls off the ordered goods as soon as they are available. Formally, in each period $t$, the buyer must call off the amount that has been ordered and actually produced, which is calculated as:

$$
x_{t}^{1 R}=\min \left(\sum_{t^{\prime}=1}^{t} x_{t^{\prime}}^{1}, \sum_{t^{\prime}=1}^{t} x_{t^{\prime}}^{2}\right)-\sum_{t^{\prime}=1}^{t-1} x_{t^{\prime}}^{1 R} .
$$

Likewise, external demand is served as soon as possible:

$$
d_{t}^{R}=\min \left(\sum_{t^{\prime}=1}^{t} d_{t^{\prime}}, \sum_{t^{\prime}=1}^{t} x_{t^{\prime}}^{1 R}\right)-\sum_{t^{\prime}=1}^{t-1} d_{t^{\prime}}^{R} .
$$


The realized setup, inventory, and backlog at the buyer are also differentiated from the planned values by using an upper index $1 R$. Note that with all the approaches investigated here, the supplier is able to execute its plans, and therefore it is not required to differentiate its production plan and the realization.

Hence, the total logistic cost, $C^{1}$, incurred at the buyer and the profit, $P^{1}$, realized by the buyer can be computed by the following formulas, where the last component, $g_{t}^{2} r_{t}^{2}$, stands for the backlog compensation received from the supplier:

$$
\begin{gathered}
C^{1}=\sum_{t=1}^{T}\left(f_{t}^{1} y_{t}^{1 R}+p_{t}^{1} x_{t}^{1 R}+h_{t}^{1} s_{t}^{1 R}+g_{t}^{1} r_{t}^{1 R}-g_{t}^{2} r_{t}^{2}\right) \\
P^{1}=\sum_{t=1}^{T} q_{t}^{1} d_{t}-\sum_{t=1}^{T} q_{t}^{2} x_{t}^{1}-\sum_{t=1}^{T}\left(f_{t}^{1} y_{t}^{1 R}+p_{t}^{1} x_{t}^{1 R}+h_{t}^{1} s_{t}^{1 R}+g_{t}^{1} r_{t}^{1 R}-g_{t}^{2} r_{t}^{2}\right)
\end{gathered}
$$

Similarly, the supplier's total cost and profit is defined by the following formulas:

$$
\begin{gathered}
C^{2}=\sum_{t=1}^{T}\left(f_{t}^{2} y_{t}^{2}+p_{t}^{2} x_{t}^{2}+h_{t}^{2} s_{t}^{2}+g_{t}^{2} r_{t}^{2}\right) \\
P^{2}=\sum_{t=1}^{T} q_{t}^{2} x_{t}^{1}-\sum_{t=1}^{T}\left(f_{t}^{2} y_{t}^{2}+p_{t}^{2} x_{t}^{2}+h_{t}^{2} s_{t}^{2}+g_{t}^{2} r_{t}^{2}\right)
\end{gathered}
$$

Finally, the complete flow of financial assets in the problem is displayed graphically in Figure 1.

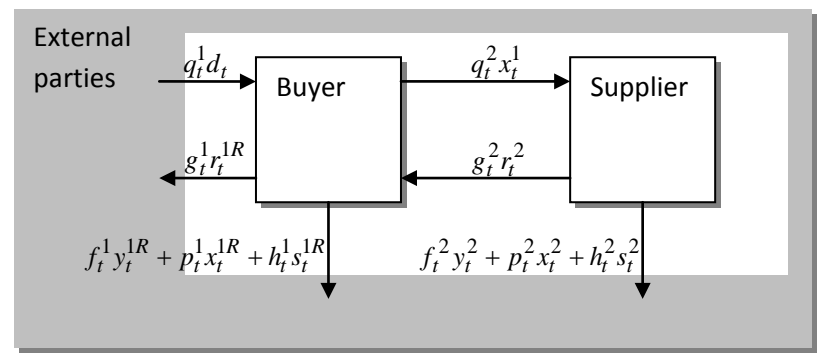

Figure 1: Flow of financial assets in the problem.

\subsection{Sample problem}

Each of the investigated approaches will be illustrated on the following sample problem instance. In this simplified example, all cost parameters are timeinvariant.

$q^{1}=\$ 14 /$ unit $\quad f^{1}=\$ 100 \quad p^{1}=\$ 1 /$ unit $\quad h^{1}=\$ 6 /$ unit/week $\quad g^{1}=\$ 18 /$ unit $/$ week

$q^{2}=\$ 8 /$ unit $\quad f^{2}=\$ 492 \quad p^{2}=\$ 1 /$ unit $\quad h^{2}=\$ 5 /$ unit/week $\quad g^{2}=\$ 6 /$ unit/week 


$$
T=10 \text { weeks } \quad d=[71,84,43,21,4,81,59,44,32,46] \text { units }
$$

\section{Decomposition approach}

When parties have no access to private data of the others, each party can but optimize its own production and logistics based on information locally available. This leads to the decomposition approach where, so as to satisfy external demand, decentralized decisions have to be coordinated. Typically, local planning problems are solved in a sequence, where the solution of one problem sets target for the next one. The most common procedure is upstream planning [8, 13], a hierarchical sequential decision scheme starting at the downstream party (e.g., retailer) who, after solving its own planning problem, generates demand to its supplier. In a longer chain, this protocol is repeated upstream.

In the decentralized approach the parties necessarily make, often implicitly, assumptions on the actions of the other related parties (e.g., the buyer may assume that its supplier always delivers on time). Whenever the above assumptions fail (e.g., the supplier delivers late), the realizations may deviate from the plans.

Information asymmetry and local autonomy cause together inefficiencies like acute shortage situations or excess inventories. Recently, Albrecht analyzed and classified a number of drivers that lead to sub-optimality in decentralized planning [2]. In any case, satisfying the target set by one partner incurs some extra costs (by, e.g., too large quantities or too frequent deliveries required) at another one, thus increasing the system-wide costs. To compensate for this deficiency, the key practical advantages of the decomposition approach are its moderate information requirements and compliance with the usual business conditions, as well as the well established theoretical and computational background.

\subsection{Computational model}

In the decomposed model of our sample problem, the buyer makes the assumption that the supplier will deliver on time (and therefore it does not hold any buffer stock), though there is no guarantee that this assumption will be satisfied in the realized scenario. Since the external demand is known by the buyer, the upstream planning approach is taken: (1) the buyer decides about its supply, inventory and backlog quantities; (2) the supply requests, $x_{t}^{1}$, are passed to the supplier as target; (3) the supplier regards these quantities as incoming demand, and computes the corresponding production plan; (4) knowing the $x_{t}^{2}$ production quantities, the delivery from the supplier to the buyer, and from the buyer to the external customer are realized according to the rules of the plan execution.

The decomposition method involves solving two identically structured singlestage lot-sizing problems, as defined by the following mixed-integer linear program (MIP). The buyer's (supplier's) model can be received by substituting $k=1(k=2)$. 
Maximize

$$
\sum_{t=1}^{T} q_{t}^{k} d_{t}^{k}-\sum_{t=1}^{T} q_{t}^{k+1} x_{t}^{1}-\sum_{t=1}^{T}\left(f_{t}^{k} y_{t}^{k}+p_{t}^{k} x_{t}^{k}+h_{t}^{k} s_{t}^{k}+g_{t}^{k} r_{t}^{k}\right) .
$$

subject to

$$
\begin{array}{ll}
x_{t}^{k}+\left(r_{t}^{k}-r_{t-1}^{k}\right)=d_{t}^{k}+\left(s_{t}^{k}-s_{t-1}^{k}\right) & t=1, \ldots, T \\
x_{t}^{k} \leq D y_{t}^{k} & t=1, \ldots, T \\
s_{0}^{k}=s_{T}^{k}=r_{0}^{k}=r_{T}^{k}=0 & \\
x_{t}^{k}, r_{t}^{k}, s_{t}^{k} \geq 0 & t=1, \ldots, T \\
y_{t}^{k} \in\{0,1\} & t=1, \ldots, T
\end{array}
$$

The MIP model maximizes the profit of the corresponding party, with $d_{t}^{1}=$ $d_{t}, d_{t}^{2}=x_{t}^{1}(7)$. The supplier $(k=2)$ does not pay any purchase price, hence $q_{t}^{k+1}=q_{t}^{3}=0$ must be considered in the objective function. Equation (8) describes the inventory balance constraint, while inequality (9) states that a positive amount can be delivered/produced in a given time period only if a setup is performed in that period. Constant $D$ is the total demand, i.e., $D=\sum_{t=1}^{T} d_{t}$. Constraints (10) set the initial and final stock and backlog to zero, which also implies that the total demand will be satisfied throughout the planning horizon. This lot-sizing problem can be solved in polynomial time, see, e.g., [18]. The buyer directly faces the external demand, i.e., $d_{t}^{1}=d_{t}(t=1, \ldots, T)$. Given that decisions on the supply requests at the buyer have already been made, the two serial decision problems are coupled: $d_{t}^{2}=x_{t}^{1}$ for each period over the horizon.

After solving the supplier's problem, from values of $x_{t}^{2}$ the realized delivery $x_{t}^{1 R}$ and the served demand $d_{t}^{R}$ can be determined according to the rules of plan execution (see (1) and (2)). The realization will incur profits $P_{D e c}^{1}$ and $P_{D e c}^{2}$, as well as costs $C_{D e c}^{1}$ and $C_{D e c}^{2}$ at the buyer and the supplier, respectively.

\subsection{Sample problem}

The solution of the sample problem computed according to the decomposition approach is displayed in Table 2 . The buyer plans to satisfy demand from justin-time supply always except for week 5 , where it wishes to use the quantity on stock from week 4. However, the supplier, who has much higher setup cost, produces only in weeks 2,6 , and 9 , which causes backlogs in weeks 1 and 8 in both the supplier-buyer and the buyer-external customer relations. This causes excess cost for the buyer compared to its plan.

\section{Integrated approach}

The inevitable sub-optimality of the decomposition approach motivated research in integrated approaches to planning in the supply chain $[1,11]$. In any case, the 


\begin{tabular}{|c|c|c|c|c|c|c|c|c|c|c|c|}
\hline & $t$ & 1 & 2 & 3 & 4 & 5 & 6 & 7 & 8 & 9 & 10 \\
\hline External demand & $d_{t}$ & 71 & 84 & 43 & 21 & 4 & 81 & 59 & 44 & 32 & 46 \\
\hline Supply request & $x_{t}^{1}$ & 71 & 84 & 43 & 25 & & 81 & 59 & 44 & 32 & 46 \\
\hline Production plan & $x_{t}^{2}$ & & 223 & & & & 140 & & & 122 & \\
\hline Delivery to buyer & $x_{t}^{1 R}$ & & 155 & 43 & 25 & & 81 & 59 & & 76 & 46 \\
\hline External demand served & $d_{t}^{R}$ & & 155 & 43 & 21 & 4 & 81 & 59 & & 76 & 46 \\
\hline
\end{tabular}

Table 2: Solution of the sample problem according to the decomposition approach. All quantities are measured in units, while profits and costs in dollars.

integrated approach presumes a central agency that knows all the parameters and whose decisions are adopted by all partners. It maximizes the total profit on the supply chain level, while, at the same time, may increase or decrease the profits of the individual parties. Compared to the decomposition approach, now the supplier is usually better off, since its parameters are also considered right at the outset of planning. To guarantee that integrated planning is beneficial for both parties, its practical implementation often involves some settlement on the sharing of profits, which may range from the reduced unit purchase prices to complex pricing schemes.

\subsection{Computational model}

Integrated planning implies that the demand set by the buyer equals the demand and the output of the supplier, i.e., $d_{t}^{2}=x_{t}^{1}$, and the supplier's backlog $r_{t}^{2}$ is zero. Consequently, the MIP model of the integrated approach corresponds to the duplication of the single-level MIP model:

Maximize

$$
\sum_{t=1}^{T} q_{t}^{1} d_{t}-\sum_{t=1}^{T}\left(f_{t}^{1} y_{t}^{1}+p_{t}^{1} x_{t}^{1}+h_{t}^{1} s_{t}^{1}+g_{t}^{1} r_{t}^{1}+f_{t}^{2} y_{t}^{2}+p_{t}^{2} x_{t}^{2}+h_{t}^{2} s_{t}^{2}\right)
$$

subject to

(8) to (12) for $k \in\{1,2\}$

The objective function contains the purchase price received from the external party, minus the total production and logistic cost in the supply chain (13). Note that the optimal solution does not contain supplier backlog, and therefore all occurrences of variables $r_{t}^{2}$ can be dropped. The constraints can be derived by substituting both $k=1$ and $k=2$ into constraints (8-12), and unifying $d_{t}^{2}$ and $x_{t}^{1}$.

We investigate the integrated approach with two different benefit sharing mechanisms. In the first case, each party bears its own costs, and hence, the profits $P_{I n t}^{1}$ and $P_{I n t}^{2}$, as well as costs $C_{I n t}^{1}$ and $C_{I n t}^{2}$ can be calculated according 
to formulas (3-6). In the second case, it is assumed that the parties share the gain over the decomposition approach, $G=P_{I n t}^{1}+P_{I n t}^{2}-P_{D e c}^{1}-P_{D e c}^{2}$, on a $50 \%-50 \%$ basis. Note that the assumptions allow the parties to compute $P_{D e c}^{1}$ and $P_{D e c}^{2}$. Finally, the shared profits are computed as $P_{I n t}^{1}{ }^{*}=P_{D e c}^{1}+\frac{G}{2}$ and $P_{I n t}^{2}{ }^{*}=P_{D e c}^{2}+\frac{G}{2}$.

\subsection{Sample problem}

The integrated solution for the sample problem of Section 2.3 is displayed in Table 3. The solution is structurally different from the decomposed solution. Since the difference between the holding costs $h^{1}$ and $h^{2}$ is marginal, the items produced at the supplier (in weeks 1,2,6, and 8) are immediately delivered to the buyer. According to this plan, the external demand will be satisfied on time, except for week 5 , where it will be backlogged. The profits realized by the individual parties increase by $7.5 \%$ (buyer) and $497.1 \%$ (supplier), which means a $216.4 \%$ improvement for the overall supply chain.

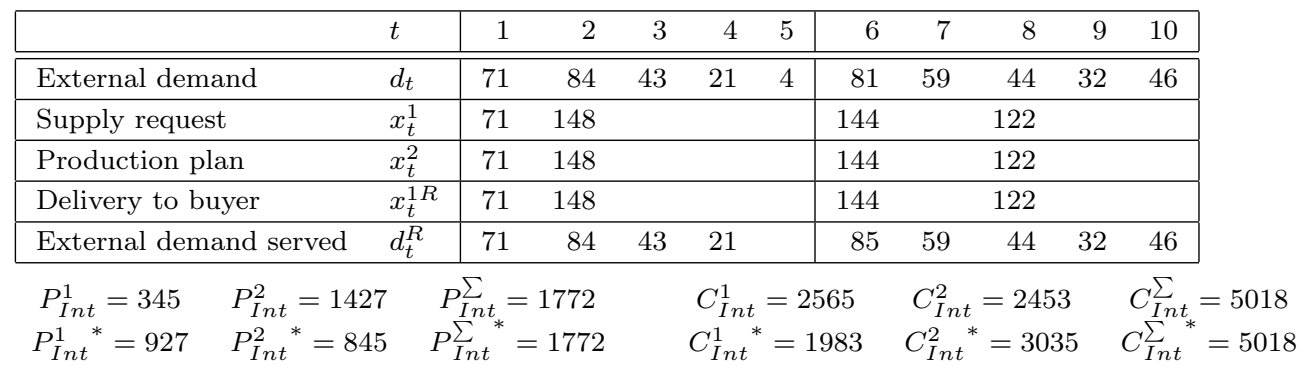

Table 3: Solution of the sample problem according to the integrated approach. All quantities are measured in units, while profits and costs in dollars.

\section{Coordinated approach}

Coordinated planning is aimed at circumventing the deficiencies of the decomposition approach when there is no opportunity for integrated planning. It strives to improve the overall performance of the supply chain while maintaining the information asymmetry and local decision authority of the partners $[2,11,16]$. A supply chain is strongly coordinated if and only if the partners' optimal local decisions lead to optimal system-wide performance. This problem can be captured in a game theoretic setting: how to find a set of optimal supply chain actions (i.e., production and delivery) that result in such an equilibrium from which no partner has an interest to deviate? The game theoretic perspective leads to theoretical contract models that coordinate a supply channel under rigorous simplifying assumptions (e.g., typically, one-period models are handled) $[5,10]$. 
In this paper, we take a weaker, albeit widely accepted concept: the supply chain is coordinated if the local, selfish production and delivery actions result in an overall performance at least as good as the decomposition approach. This definition allows for a wide spectrum of coordination mechanisms that have though some generic requirements in common: (1) while keeping the privacy of sensitive cost and profit factors, there is a need for sharing the intentionsspecifically, plans-of the partners; (2) alternative planning scenarios have to be generated, exchanged and evaluated; (3) an incentive scheme is required thatagainst their local interests-drives the partners towards coordinated solutions. Hence, potential benefits of coordination should be shared.

\subsection{Computational model}

Our model for coordinated planning builds on the elements of the decomposed model. Instead of a single production plan, the supplier responds to the demand of the buyer with a set of alternative scenarios from among which the buyer will finally select a single one to be executed. However, compared to its optimal production plan, the supplier can only have a loss on each of the alternative scenarios, hence, it assigns a compensation request to each of them. The alternative planning scenarios are distinguished by index $n=0, \ldots, N$, and the costs and profits of a particular scenario from the perspective of the buyer and the supplier are expressed by $C^{1, n}, C^{2, n}, P^{1, n}$ and $P^{2, n}$, respectively. Locally optimal solutions of the decomposed approach are indexed by $n=0$.

The coordinated planning protocol proceeds in the following steps: (1) The buyer solves its problem as formulated in equations (7)-(12), facing directly the external demand, i.e., $d_{t}^{1}=d_{t},(t=1, \ldots, T)$. (2) The supply requests $x_{t}^{1}$ are communicated to the supplier who in return generates a baseline production plan $x_{t}^{2,0}$, incurring profit $P^{2,0}$. With some policy (discussed later) the supplier generates a series of alternative production plans, $x_{t}^{2, n}$. The supplier's potential loss on each scenario is calculated as $L^{2, n}=P^{2,0}-P^{2, n}$. (3) The alternative scenarios are offered to the buyer, together with a compensation requirement $Z^{2, n}$. Obviously, the self-interested supplier will ask for a compensation that covers its loss, i.e., $Z^{2, n} \geq L^{2, n}$. (4) The buyer simulates the execution of each $x_{t}^{2, n}$ scenario received, and calculates the profit $P^{1, n}$ as the realized profit given in (4) minus the compensation $Z^{2, n}$. (5) Finally, the buyer selects the scenario with the maximal profit $P^{1, n}$ and the chain as a whole will be operated accordingly.

Since $Z^{2, n} \geq L^{2, n}$, the partners deviate from the baseline solution of the decomposition approach only if there is a scenario $n$ where $P^{1, n}+P^{2, n}>P^{1,0}+$ $P^{2,0}$. In the last resort, the baseline solution is executed. Hence, the above protocol coordinates the chain in the weak sense.

The policy for scenario generation is based on the idea that not only the buyer, but also the chain as a whole could be better off if the buyer did not have to face backlog. Hence, the supplier generates a series of alternative scenarios with less and less backlog, ending up with a production plan scenario $N$ without any backlog at the supplier (i.e., in every period $t, r_{t}^{2, N}=0$ ). Such a series 
can be generated by using forged, increased backlog cost parameters $g_{t}^{2, n}$ for computing the plans. We have used parameters $g_{t}^{2, n+1}=1.1 g_{t}^{2, n}$ with $g_{t}^{2,0}=g_{t}^{2}$. Since the supply requests are generated a priori, the proposed alternatives may not contain the optimal solution of the integrated approach.

In our model the benefit of eventual cooperation can be shared through the compensation required by the supplier for a sub-optimal scenario. A supplier with a fully cooperative but rational attitude does not require more than its eventual loss, i.e., $Z^{2, n}=L^{2, n}$. Though, in addition to this the supplier may want to realize some gain, too. The gain ratio $\varrho$ is given as the percentage of the cost in the baseline solution, resulting in a compensation of $Z^{2, n}=L^{2, n}+\varrho C^{2,0}$. If the supplier has a greedy attitude and requires more compensation than its potential loss, then the chances of arriving at a coordinated solution are getting worse.

Finally, it must be noted that in contrast to the previous approaches, the solution computed by the coordination approach is characteristic to the defined coordination mechanism; different mechanisms may result in different solutions. A shortfall of this coordination mechanism is that the supplier, if it has information about the buyer's parameters, can abuse the mechanism: it can deliberately generate a baseline plan that is unacceptable to the buyer, and assign massive compensation costs to any other alternatives.

\subsection{Sample problem}

Using the above mechanism with $\varrho=0$, a series of production plan alternatives have been generated for the sample problem. These contained the baseline decomposed solution and additional four alternative production plans for the supplier. The last alternative, with modified backlog cost $g^{2,4}=9.663$, resulted in no backlog in either relation. This alternative incurs a higher cost of 3833 for the supplier (cf. the default $C_{D e c}^{2}=3641$ ). However, it is worth for the buyer to compensate the supplier for eliminating the costly backlog, and hence, this plan alternative is selected. This solution increases the profit of the overall supply chain by $176.4 \%$ compared to the decomposition approach, but is still inferior to the integrated solution, because the supply requests of the buyer, computed a priori, did not enable the partners to find a more efficient solution.

\section{Bilevel approach}

The bilevel optimization approach captures the decision situation of a wellinformed buyer (leader in the terminology of bilevel optimization), who knows the decision problem of the supplier (follower), i.e., the parameters $f_{t}^{2}, p_{t}^{2}, h_{t}^{2}$ and $g_{t}^{2}$, and wants to take into account the optimal decision of the supplier when computing its supply requests. We adopt the optimistic assumption, i.e., consider that in case of multiple optimal solutions for the supplier, it chooses the optimal solution which is the most favorable for the buyer. Notice that in 


\begin{tabular}{|ll|rrrrr|rrrrr|}
\hline & $t$ & 1 & 2 & 3 & 4 & 5 & 6 & 7 & 8 & 9 & 10 \\
\hline \hline External demand & $d_{t}$ & 71 & 84 & 43 & 21 & 4 & 81 & 59 & 44 & 32 & 46 \\
\hline Supply request & $x_{t}^{1}$ & 71 & 84 & 43 & 25 & & 81 & 59 & 44 & 32 & 46 \\
\hline Production plan & $x_{t}^{2}$ & 71 & 152 & & & & 140 & & 122 & & \\
\hline Delivery to buyer & $x_{t}^{1 R}$ & 71 & 84 & 43 & 25 & & 81 & 59 & 44 & 32 & 46 \\
\hline External demand served & $d_{t}^{R}$ & 71 & 84 & 43 & 21 & 4 & 81 & 59 & 44 & 32 & 46 \\
\hline & $P_{C r d}^{1}=1309 \quad P_{C r d}^{2}=239$ \\
\hline
\end{tabular}

Table 4: Solution of the sample problem according to the coordinated approach, assuming a fully cooperative supplier, i.e., the gain ratio set to zero. All quantities are measured in units, while profits and costs in dollars.

the pessimistic case the supplier would always choose an optimal solution which yields the least favorable outcome for the buyer.

The basic modeling and solution techniques in bilevel programming are presented in [7]. However, the literature of bilevel approaches to inventory problems is rather scarce. The most relevant references include [6] and [14], where two different production and delivery planning problems are investigated in extended supply chain, with the goal of constructing plans that are locally optimal for the individual parties as well. In [17], the problem of coordinated planning in a supply chain under hard service time requirements is investigated, where a central coordinating agency allocates desired response times to the individual parties.

\subsection{Computational model}

In the following mathematical program we model the decision problem of the leader. The decision variables and parameters are similar to the ones in the previous approaches.

Maximize

$$
\sum_{t=1}^{T} q_{t}^{1} d_{t}-\sum_{t=1}^{T} q_{t}^{2} x_{t}^{1}-\sum_{t=1}^{T}\left(f_{t}^{1} y_{t}^{1}+p_{t}^{1}\left(x_{t}^{1}+r_{t-1}^{2}-r_{t}^{2}\right)+h_{t}^{1} s_{t}^{1}+g_{t}^{1} r_{t}^{1}-g_{t}^{2} r_{t}^{2}\right)
$$

subject to

$$
\begin{array}{ll}
x_{t}^{1}+r_{t-1}^{2}-r_{t}^{2}+\left(r_{t}^{1}-r_{t-1}^{1}\right)=d_{t}^{1}+\left(s_{t}^{1}-s_{t-1}^{1}\right) & t=1, \ldots, T \\
x_{t}^{1}+r_{t-1}^{2}-r_{t}^{2} \leq D y_{t}^{1} & t=1, \ldots, T
\end{array}
$$

(10) to (12) with $k=1$ 


$$
\left(\begin{array}{c}
y_{t}^{2} \\
x_{t}^{2} \\
s_{t}^{2} \\
r_{t}^{2}
\end{array}\right) \in \arg \max \left\{\sum_{t=1}^{T} q_{t}^{2} x_{t}^{1}-\sum_{t=1}^{T}\left(f_{t}^{2} y_{t}^{2}+p_{t}^{2} x_{t}^{2}+h_{t}^{2} s_{t}^{2}+g_{t}^{2} r_{t}^{2}\right) \mid(8) \text { to (12) with } k=2\right\}
$$

The objective (14) is maximizing the leader's profit, considering purchase prices received and paid, all logistic costs, as well as the compensation received from the supplier for late deliveries. The constraints of the model are similar to those in the previous approaches, except that the leader must calculate with the realized supply $x_{t}^{1 R}=x_{t}^{1}+r_{t-1}^{2}-r_{t}^{2}$, which may differ from the supply request submitted, $x_{t}^{1}$. Otherwise, the constraints of both the buyer and the supplier are identical to those in the decomposed model. The supplier's optimality condition (18) expresses that the supplier chooses its optimal production plan for the given supply requests received from the buyer. Again, $d_{t}^{2}$ must be substituted with $x_{t}^{1}$.

It must be noted that there exists no generic solution method for mixed discrete-continuous bilevel optimization problems such as the bilevel lot-sizing problem under consideration. Instead, we developed a customized solution algorithm, motivated by the dynamic program (DP) of Zangwill [18] for the singlestage uncapacitated lot-sizing problem with backlogs. The DP exploits that the single-level problem (and hence, the supplier's subproblem in our bilevel setting) always admits an optimal solution that consists of so-called regeneration intervals. The ensemble of such regeneration intervals is called a configuration. The algorithm decomposes the problem according to the possible configurations of the supplier's production plan. For each configuration, it solves a single-level MIP with added constraints that a production plan with the given configuration must be optimal for the supplier. The detailed presentation of the algorithm can be found in Appendix A.

\subsection{Sample problem}

The solution of the sample problem according to the bilevel approach is shown in Table 5. It demonstrates the various ways how the buyer can manipulate the supply requests submitted to the supplier so as to minimize its own cost. Namely, in week 1, the buyer asks for a larger lot than its actual needs ( 82 units instead of 71). This is necessary in order to prevent the supplier from backlogging this lot to week 2 (cf. the decomposed solution in Table 2), which would cause an extensive backlog cost for the buyer as well. On the other hand, the buyer anticipates some demand from week 6 to week 5 . The supply request for week 5 is then the maximum amount that does not trigger production at the supplier. While this kind of demand anticipation does not affect the material flow, it incurs a backlog compensation paid by the supplier to the buyer for the late satisfaction of the anticipated demand. Certainly, this can be regarded as an abuse of the contract between parties, but this is a rational action from a profit maximizing buyer. 
Compared to the decomposition approach, the buyer could increase its profit by $384.8 \%$, but this also led to a moderate, $6.9 \%$ increase of the costs of the supplier; finally, in this problem instance, the supplier's profit turned negative. The overall profit in the supply chain increased by $175.5 \%$. Note that in general there is no guarantee that the bilevel approach increases the total profit.

\begin{tabular}{|lc|rrrrr|rrrrr|}
\hline & $t$ & 1 & 2 & 3 & 4 & 5 & 6 & 7 & 8 & 9 & 10 \\
\hline \hline External demand & $d_{t}$ & 71 & 84 & 43 & 21 & 4 & 81 & 59 & 44 & 32 & 46 \\
\hline Supply request & $x_{t}^{1}$ & 82 & 73 & 68 & & 42.72 & 39.77 & 57.51 & 55.46 & 21.93 & 44.61 \\
\hline Production plan & $x_{t}^{2}$ & 82 & 141 & & & & 140 & & 122 & & \\
\hline Delivery to buyer & $x_{t}^{1 R}$ & 82 & 73 & 68 & & & 82.49 & 57.51 & 55.46 & 21.93 & 44.61 \\
\hline External demand served & $d_{t}^{R}$ & 71 & 84 & 43 & 21 & 4 & 81 & 59 & 44 & 32 & 46 \\
\hline & \multirow{2}{*}{$P_{B l}^{1}=1555.36$} & $P_{B l}^{2}=-12.69$ & \multicolumn{2}{|c}{$P_{B l}^{\sum}=1542.67$} & \multicolumn{2}{|c}{$C_{B l}^{1}=1354.64$} & $C_{B l}^{2}=3892.69$ & $C_{B l}^{\sum}=5247.33$
\end{tabular}

Table 5: Solution of the sample problem according to the bilevel approach. All quantities are measured in units, while profits and costs in dollars.

\section{Comparison of the approaches}

\subsection{Comparison of requirements}

After the detailed presentation of the individual approaches, we compare their requirements and applicability to various scenarios in the supply chain analytically in Table 6 . The aspects of the comparison are defined below.

- Information requirements: what information has to be shared among the different parties?

- Cooperation: what kind of cooperation is required among the parties?

- Extra contractual requirements: what aspects of the cooperation have to be laid down in contracts, in addition to the common costs, dates, and conditions?

- Applicability to multi-level: is the approach applicable to multi-level supply chains? What are the limitations of this extension?

- Optimization: what optimization criterion does the approach address?

- Computational tractability: what computational challenges does the approach pose in a typical application? 


\begin{tabular}{|c|c|c|c|c|}
\hline & Decomposition & Integrated & Coordinated & Bilevel \\
\hline $\begin{array}{l}\text { Information } \\
\text { requirements }\end{array}$ & $\begin{array}{l}\text { Buyer commu- } \\
\text { nicates demand } \\
\text { only }\end{array}$ & $\begin{array}{l}\text { Planner has full in- } \\
\text { formation on all } \\
\text { parties }\end{array}$ & $\begin{array}{l}\text { Demand, alterna- } \\
\text { tive plans, compen- } \\
\text { sation costs com- } \\
\text { municated }\end{array}$ & $\begin{array}{l}\text { Buyer has full in- } \\
\text { formation on the } \\
\text { supplier; supplier } \\
\text { receives demand }\end{array}$ \\
\hline Cooperation & Selfish parties & Full cooperation & Selfish parties & Selfish parties \\
\hline $\begin{array}{l}\text { Extra con- } \\
\text { tractual req. }\end{array}$ & - & $\begin{array}{l}\text { Rules of informa- } \\
\text { tion and benefit } \\
\text { sharing }\end{array}$ & $\begin{array}{l}\text { Rules of informa- } \\
\text { tion and benefit } \\
\text { sharing }\end{array}$ & - \\
\hline $\begin{array}{l}\text { Applicability } \\
\text { to multi-level }\end{array}$ & Yes & $\begin{array}{l}\text { Yes, with limita- } \\
\text { tions (data accessi- } \\
\text { bility, sensitivity to } \\
\text { disturbances). }\end{array}$ & $\begin{array}{l}\text { Yes, with limita- } \\
\text { tions on the appli- } \\
\text { cable coordination } \\
\text { mechanisms }\end{array}$ & Impracticable \\
\hline Optimization & - & $\begin{array}{l}\text { Supply chain total } \\
\text { profit }\end{array}$ & $\begin{array}{l}\text { Supply chain total } \\
\text { profit (with restric- } \\
\text { tions by the mech- } \\
\text { anism applied) }\end{array}$ & Buyer's profit \\
\hline $\begin{array}{l}\text { Computational } \\
\text { tractability }\end{array}$ & $\begin{array}{l}\text { Local problems } \\
\text { solved (often } \\
\text { tractable) }\end{array}$ & $\begin{array}{lr}\text { Integrated } & \text { prob- } \\
\text { lems } & \text { solved } \\
\text { (tractable } & \text { cases } \\
\text { exist) } & \end{array}$ & $\begin{array}{l}\text { Series of local prob- } \\
\text { lems solved (often } \\
\text { tractable) }\end{array}$ & $\begin{array}{l}\text { Embedded op- } \\
\text { timization (ex- } \\
\text { tremely challeng- } \\
\text { ing) }\end{array}$ \\
\hline
\end{tabular}

Table 6: Analytical comparison of the different approaches.

\subsection{Comparison of solutions, profits, and costs}

The solutions delivered by the different approaches have been compared quantitatively in computational experiments on a set of randomly generated problem instances. For that purpose, the presented models and algorithms have been implemented in FICO XPress-MP [9], using the Mosel programming language. In the numerical study, 100 problem instances were generated with the following parameters. The number of time periods was fixed to $T=10$, and all cost parameters were assumed to be constant over time. Furthermore, fixed parameters $f^{1}=100$ and $p_{t}^{1}=p_{t}^{2}=0$ were considered in all instances, while the other cost parameters were randomized. In the sequel, $U[a, b]$ stands for the uniform distribution over the integers in interval $[a, b]$. Hence, we let $h^{1} \leftarrow U[2,10]$, $g^{1} \leftarrow U[4,20], f^{2} \leftarrow U[250,500], h^{2} \leftarrow U\left[1, h^{1}\right]$, and $g^{2} \leftarrow U\left[2, g^{1}\right]$. The per unit purchase prices, $q_{t}^{1}$ and $q_{t}^{2}$, were chosen to ensure a reasonable profit for the parties, by using an Economic Order Quantity (EOQ) formula to estimate the costs and applying a $10 \%$ profit margin.

We recall that for the decomposed, integrated, and bilevel approaches, optimal solutions of formal mathematical models were computed (in case of the decomposed approach, the typical upstream planning method was considered). In contrast, the solution found by the coordinated approach was characteristic to the developed coordination mechanism; different mechanisms may lead to different solutions. Finding the optimal solutions required a few minutes per instance for the bilevel solver, while running times were negligible for the other three solvers.

The results are displayed in Figures 2 to 4, which compare the profits and 
costs incurred according to the integrated, coordination, and bilevel approaches, respectively, to the values computed according to the baseline decomposition approach. Each spot in a diagram corresponds to one problem instance, and its horizontal (vertical) position shows the difference of the buyer's (supplier's) profit or cost according to the two compared approaches. More precisely, in the figures presenting profits, the coordinates of a point correspond to the improvement of the profit margins, i.e.,

$$
\left[100\left(\frac{P_{X}^{1}}{R}-\frac{P_{D e c}^{1}}{R}\right), 100\left(\frac{P_{X}^{2}}{R}-\frac{P_{D e c}^{2}}{R}\right)\right],
$$

where $P_{X}^{k}$ stands for the profit realized by party $k$ according to approach $X$, and $R=\sum_{t=1}^{T} q_{t}^{1} d_{t}$ is the revenue of overall supply chain. The upper right quarter of the diagrams contains the solutions that are beneficial for both parties, whereas the lower right quarter contains solutions advantageous for the buyer, but disadvantageous for the supplier, etc. A spot above the diagonal denotes an instance where the investigated approach could increase the overall profit of the supply chain compared to the decomposition approach. In figures comparing costs, the coordinates of the spots are

$$
\left[100\left(\frac{C_{X}^{1}}{C_{D e c}^{\sum}}-\frac{C_{D e c}^{1}}{C_{D e c}^{\sum}}\right), 100\left(\frac{C_{X}^{2}}{C_{D e c}^{\sum}}-\frac{C_{D e c}^{2}}{C_{D e c}^{\sum}}\right)\right],
$$

i.e., the improvement is measured in percent of the overall total cost of the decomposed solution. Hence, a spot with coordinates $(-25,-15)$ denotes that the total cost was decreased by $40 \%$. Here, the lower left quarter of the diagrams contain the solutions that are beneficial for both parties. A solution below the diagonal decreases the cost of the overall supply chain.

It is emphasized that the benefits are higher compared to the profit or the cost of the individual party, by a factor of 2 on average. For example, in the solution corresponding to the above spot, $(-25,-15)$, if $C_{D e c}^{1}=C_{D e c}^{2}$, then the buyer's and the supplier's costs have decreased by $50 \%$ and $30 \%$, respectively, in the percent of the cost of the individual party. Still, the reason for applying the above measures was that in this way, the solutions neutral for the overall supply chain fall on the diagonal.

Figure 2 shows the profits and costs obtained by the integrated approach. As expected, integration increases the profit and decreases the cost of the overall supply chain for most instances (86 out of 100), by $16.54 \%$ (profit) $8.67 \%$ (cost) on average. For the remaining instances, the overall profit and cost is unchanged. Without benefit balancing, this approach is advantageous especially for the supplier, whose profit increased in 80 out of the 100 problem instances. The reason for this is that the decomposition approach completely ignores the supplier's objective in the first round of decision making. Integration can be beneficial for the buyer when centralized planning eliminates its backlog originating from the difference of the requested supply and the realized deliveries (25 instances). 

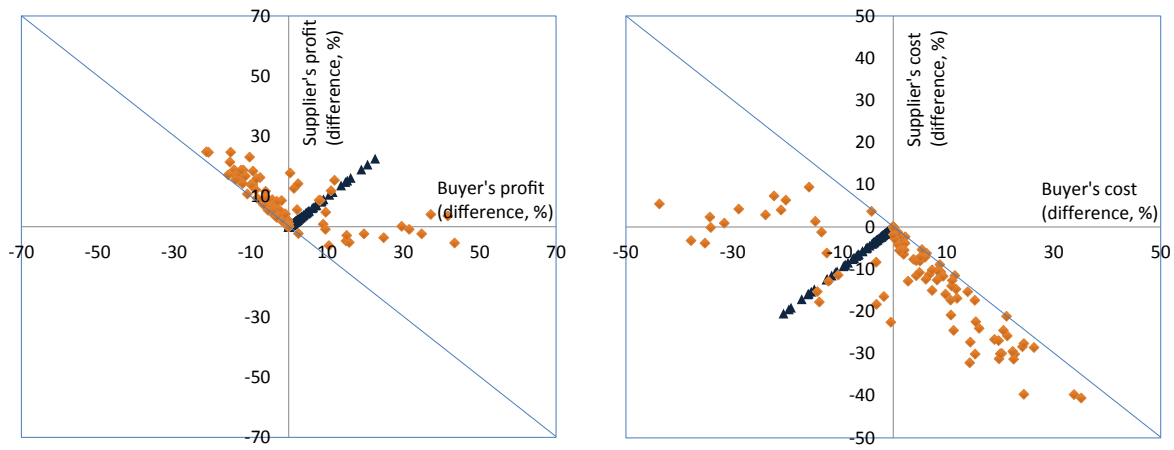

Figure 2: Comparison of the realized profits (left) and the incurred costs (right) according to the integrated and the decomposition approaches. The light (orange) diamonds correspond to solutions without benefit sharing, whereas the dark (blue) triangles stand for solutions with benefit sharing on a 50-50 basis.

The results of the coordinated approach are displayed in Figure 3. If the supplier is fully cooperative, it realizes the same profits and costs as with the decomposition approach, but the overall performance is improved for 36 out of the 100 instances, by $10.53 \%$ (profit) and $5.01 \%$ (cost) on average. With $30 \%$ supplier gain ratio, the overall performance improved for only 15 instances, by $8.05 \%$ (profit) and $3.62 \%$ (cost), but this gain is shared between the parties. A fundamental difference between the integration and the coordination approaches can be easily observed on the above diagrams: integration pursues the benefit of the overall chain, which corresponds to spots on one side of the diagonal. On the other hand, the self-interested attitude of the parties in the coordination approach leads to spots in the appropriate quarter plane in each of the diagrams.

Finally, Figure 4 contains the results of the bilevel approach. The informed buyer could increase its profit and reduce its costs in all cases, by $20.03 \%$ (profit) and $10.91 \%$ (cost) on average, compared to the unaware buyer of the decomposition case. Notably, this is a $33.89 \%$ increase of profit and a $33.54 \%$ reduction of cost, if we take the decomposed buyer's profit and cost as the basis. Bilevel planning is generally not beneficial for the supplier, whose profit decreased and costs increased for 72 instances, by $9.78 \%$ (profit) and $5.94 \%$ (cost) on average. Although there is no guarantee that bilevel optimization improves the performance of the overall supply chain, a $10.25 \%$ increase of profit and a $4.98 \%$ reduction of costs could be observed for the given set of instances. Surprisingly, this is better result than the one achieved by coordination with the supplier's gain ratio set to $30 \%$. A possible interpretation of this result is that (even asymmetric) information can reduce the overall cost more efficiently than the incentive to cooperate without sufficient information. 

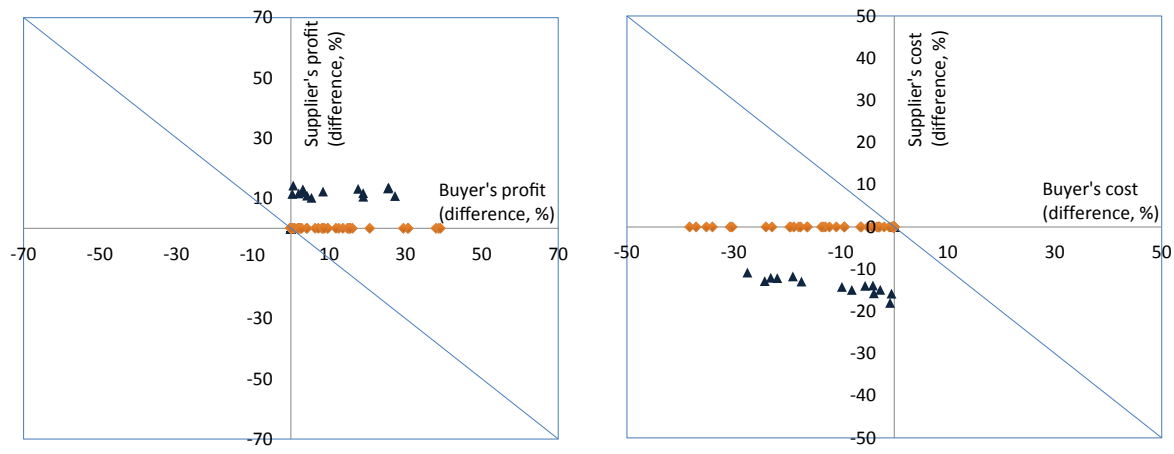

Figure 3: Comparison of the realized profits (left) and the incurred costs (right) according to the coordinated and the decomposition approaches. The light (orange) diamonds represent the case with a fully cooperative supplier (gain ratio set to zero), while the dark (blue) triangles correspond to the case with the gain ratio of $30 \%$.
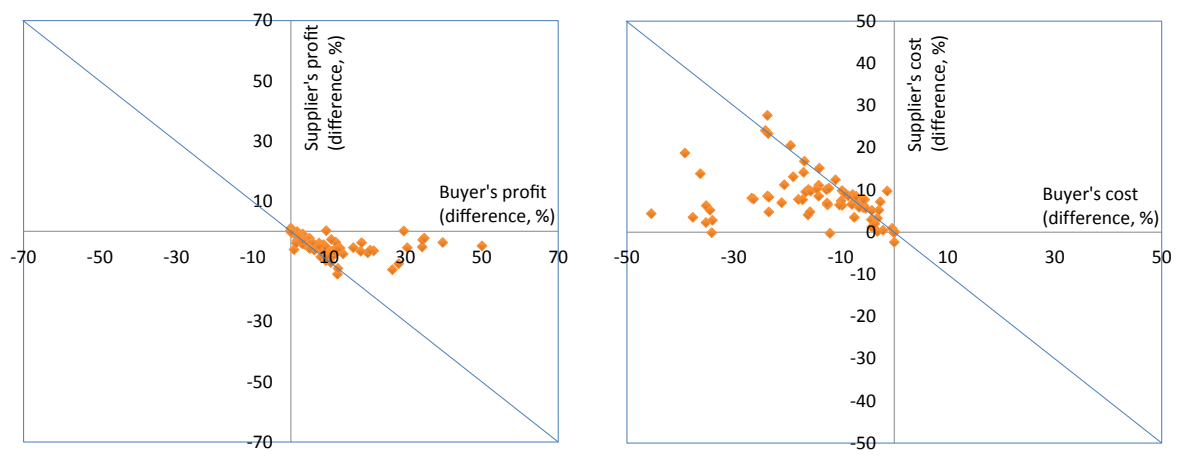

Figure 4: Comparison of the realized profits (left) and the incurred costs (right) according to the bilevel and the decomposition approaches.

\section{Conclusions}

This paper investigated four different computational approaches to solving the same lot-sizing problem in a supply chain consisting of two parties. The comparison focused on how each of the approaches handles the aspects of autonomy, information asymmetry, and conflicting objectives. Moreover, to compare the incurred profits and costs for the different parties, algorithms have been implemented for solving the analyzed problem according to each of the four approaches. This also required the development of a new coordination mechanism for the coordinated approach, as well as a new exact algorithm for the bilevel approach.

Certain findings of this study are relevant beyond the scope of the specific lot-sizing problem as well. Especially, it has been demonstrated that for a given 
inventory control problem, the profits incurred for the different parties extraordinarily depend on the applied solution approach: e.g., the buyer could increase its profit by ca. $34 \%$ on average, if in the possession of sufficient information, it switched from a decomposition strategy to bilevel optimization. Also, it has been emphasized that such novel approaches as coordination or bilevel optimization are applicable to lot-sizing problems, and they can provide additional benefits for the parties in the supply chain. In particular, the applicability of the classical integrated approach is limited to cases where the business objectives of the parties are completely aligned and they are ready to share all relevant data with each other. In case of autonomous parties with disparate objectives, a coordination approach may bring comparable savings, if the dynamics of the chain (both in terms of stable network design and non-critical response times) allow for appropriate contracts and communication mechanisms. On the other hand, an individual party, having access to sufficiently precise data about its upstream partners, may optimize its own production or logistics by implementing a bilevel optimization approach.

\section{Acknowledgements}

This work has been supported by the OTKA grant K76810, the NKTH grant OMFB-01638/2009, as well as TÁMOP-4.2.1/B-09/1/KMR-2010-0002 grant. A. Kovács and P. Egri acknowledge the support of the János Bolyai scholarship no. BO/00138/07 and no. BO/00659/11/6.

\section{References}

[1] B. Abdul-Jalbar, J. Gutiérrez, J. Puerto, and J. Sicilia. Policies for inventory/distribution systems: The effect of centralization vs. decentralization. International Journal of Production Economics, 81-82:281-293, 2003.

[2] M. Albrecht. Supply Chain Coordination Mechanisms. Springer, 2010.

[3] S. Axsäter. Inventory Control. Springer, 2nd edition, 2006.

[4] J. F. Bard. Coordination of a multidivisional organization through two levels of management. Omega, 11:457-468, 1983.

[5] G.P. Cachon. Supply Chain Management: Design, Coordination and Cooperation, chapter Supply chain coordination with contracts, pages 229-339. Elsevier, 2003.

[6] A. G. de Kok and G. Muratore. Coordinating supply chains: a bilevel programming approach. In International Workshop on Supply Chain Models for Shared Resource Management, 2010.

[7] S. Dempe. Foundations of Bilevel Programming. Kluwer, 2002. 
[8] G. Dudek and H. Stadtler. Negotiations-based collaborative planning between supply chain partners. European Journal of Operational Research, 163:668-687, 2005.

[9] FICO. Xpress Optimization Suite 7. http://www.fico.com/en/Products/ DMTools/Pages/FICO-Xpress-Optimization-Suite.aspx.

[10] M. Leng and M. Parlar. Game theoretic applications in supply chain management: a review. INFOR, 43(3):187-220, 2005.

[11] X. Li and Q. Wang. Coordination mechanisms of supply chain systems. European Journal of Operational Research, 179(1):1-16, 2007.

[12] G. Perakis and G. Roels. The price of anarchy in supply chains: Quantifying the efficiency of price-only contracts. Management Science, 53(8):12491268, 2007.

[13] R. Pibernik and E. Sucky. An approach to inter-domain master planning in supply chains. International Journal of Production Economics, 108:200210, 2007.

[14] J-H. Ryu, V. Dua, and E. N. Pistikopoulos. A bilevel programming framework for enterprise-wide process networks under uncertainty. Computers and Chemical Engineering, 28:1121-1129, 2004.

[15] S.P. Sarmah, D. Acharya, and S.K. Goyal. Buyer vendor coordination models in supply chain management. European Journal of Operational Research, 175(1):1-15, 2006.

[16] H. Stadtler. A framework for collaborative planning and state-of-the-art. OR Spectrum, 31:5-30, 2009.

[17] W. Yang, L. Li, and S. Ma. Coordinating supply chain response-time: a bi-level programming approach. International Journal of Advanced Manufacturing Technology, 31:1034-1043, 2007.

[18] W. I. Zangwill. A backlogging model and a multi-echelon model of a dynamic economic lot size production system - a network approach. Management Science, 15(9):506-527, 1969.

\section{A Algorithm for the bilevel approach}

Below we give an exact algorithm for solving the bilevel inventory control problem defined in Section 6. The algorithm is motivated by the dynamic program (DP) of Zangwill [18] for the single-stage uncapacitated lot-sizing problem with backlogs (ULSB). This DP, with its parameters taken from the supplier's problem, is exploited to characterize the production plans that are optimal for the supplier for some specific supply request defined by the buyer. It is noted that the original formulation of the DP addresses the minimization of the (supplier's) 
total cost, which, in the investigated bilevel setting, is equivalent to maximizing its profit, since the supplier's revenue is independent of the supplier's decisions.

Recall that the single-stage ULSB problem always admits an optimal solution of the following structure [18]: there exists an integer $K \geq 1$ and a sequence of $2 K$ integers $1=\ell_{1} \leq i_{1}<\ell_{2} \leq i_{2}<\cdots<\ell_{K} \leq i_{K} \leq T$ such that $x_{j} \neq 0$ only if $j \in\left\{i_{1}, i_{2}, \ldots, i_{K}\right\}$ from which periods $\ell_{j}, \ldots, i_{j}-1$ are satisfied by backlogging, while periods $i_{j}+1, \ldots, \ell_{j}-1$ from stock, and $s_{\ell_{j}-1}=r_{\ell_{j}-1}=0$. We call these $2 K$ integers a configuration.

In order to solve the bilevel optimization problem, we will search over all possible configurations that may be implemented by the supplier. For each configuration, we derive the conditions under which the configuration may be optimal for the supplier for the demand $x_{t}^{1}$, based on the DP of Zangwill. The conditions will take the form of linear inequalities in $x_{t}^{1}$ and some extra variables. We will add them to the buyer's constraints (15)-(17), and solve the resulting MIP. Repeating this for each configuration, and taking the minimum value of the optimal solutions of the MIPs, we obtain the optimal solution of the bilevel optimization problem. It remains to derive the conditions for a demand vector $d_{t}^{2}=x_{t}^{1}$ such that a configuration is optimal for the supplier.

The dynamic program of Zangwill, formulated with the demand and costs of the supplier, is as follows. Let $\phi(u, v)$ denote the optimal solution value of the supplier provided the demand in period $v$ is satisfied from period $u$. Furthermore, let $G(v)$ denote the optimal solution value of the problem restricted to the periods $v, \ldots, T$, i.e., $G(v)=\min _{u \geq v} \phi(u, v)$. Finally, let $h_{t}^{2^{\prime}}=h_{t}^{2}+p_{t}^{2}-p_{t+1}^{2}$ $\left(p_{T+1}^{2}=0\right)$ and $g_{t}^{2^{\prime}}=g_{t}^{2}-p_{t}^{2}+p_{t+1}^{2}$. Now we can define $\phi(u, v)$ formally:

$$
\phi(u, v)= \begin{cases}\left(\sum_{t_{u}}^{v-1} h_{t}^{2^{\prime}}\right) d_{v}^{2}+\min \{G(v+1), \phi(u, v+1)\} & \text { when } u<v \\ \left(\sum_{t=v}^{u-1} g_{t}^{2^{\prime}}\right) d_{v}^{2}+\phi(u, v+1) & \text { when } u>v \\ f_{u}^{2}+\min \{G(u+1), \phi(u, u+1)\} & \text { when } u=v .\end{cases}
$$

The optimal supplier solution value is $G(1)$ which can be computed by decreasing $u$ from $T$ down to 1 and for each $u$ in turn, iterating $v$ from $T$ down to 1. Now, all we need is to use this dynamic program to express conditions under which a configuration of the supplier is optimal. The following inequalities describe a relaxation of the dynamic program: 


$$
\begin{aligned}
G(v) \leq \phi(u, v) & \text { when } u \geq v \\
\phi(u, v) \leq\left(\sum_{t=u}^{v-1} h_{t}^{2^{\prime}}\right) d_{v}^{2}+G(v+1) & \text { when } u<v \\
\phi(u, v) \leq\left(\sum_{t=u}^{v-1} h_{t}^{2^{\prime}}\right) d_{v}^{2}+\phi(u, v+1) & \text { when } u<v \\
\phi(u, v)=\left(\sum_{t=v}^{u-1} g_{t}^{2^{\prime}}\right) d_{v}^{2}+\phi(u, v+1) & \text { when } u>v \\
\phi(u, v) \leq f_{u}^{2}+G(u+1) & \text { when } u=v \\
\phi(u, v) \leq f_{u}^{2}+\phi(u, u+1) & \text { when } u=v \\
G(T+1)=\phi(u, T+1)=0 & \text { when } u \leq T \\
\phi(u, v), G(v) \geq 0 & \text { for all } u, v
\end{aligned}
$$

Lemma 1 The configuration $1=\ell_{1} \leq i_{1}<\ell_{2} \leq i_{2}<\cdots<\ell_{K} \leq i_{K} \leq T$ is optimal for demand $d_{t}^{2}$ if and only if there exists $(\phi, G)$ that satisfies (19)-(26), and also for each $j=1, \ldots, K$ :

$$
\begin{aligned}
& \text { i) } G\left(\ell_{j}\right)=\phi\left(i_{j}, \ell_{j}\right), \\
& \text { ii) } \phi\left(i_{j}, v\right)=\left(\sum_{t=i_{j}}^{v-1} h_{t}^{2^{\prime}}\right) d_{v}^{2}+\phi\left(i_{j}, v+1\right) \text { for } i_{j}<v<\ell_{j+1}-1, \\
& \text { iii) } \phi\left(i_{j}, v\right)=\left(\sum_{t=i_{j}}^{v-1} h_{t}^{2^{\prime}}\right) d_{v}^{2}+G(v+1) \text { for } v=\ell_{j+1}-1, \\
& \text { iv) } \phi\left(i_{j}, i_{j}\right)=f_{i_{j}}^{2}+\phi\left(i_{j}, i_{j}+1\right) \text { if } i_{j}<\ell_{j+1}-1, \\
& \text { v) } \phi\left(i_{j}, i_{j}\right)=f_{i_{j}}^{2}+G\left(i_{j}+1\right) \text { if } i_{j}=\ell_{j+1}-1 .
\end{aligned}
$$

Proof The crux of the proof is that we observe how the quantities $G(v)$ and $\phi(u, v)$ relate in an optimal solution with the given configuration.

Necessity: Suppose the given configuration is optimal. Since $G(v)=\min _{u \geq v} \phi(u, v)$, condition (i) is satisfied. Moreover, for $j=1, \ldots, K$, the demand $d_{\ell_{j}}, \ldots, \bar{d}_{\ell_{j+1}-1}$ is satisfied from the time period $i_{j}$, which implies conditions (ii)-(v).

Sufficiency: We have to prove that the configuration is optimal, provided there exist $\phi(u, v)$ and $G(v)$ satisfying the conditions (i)-(v) along with (19)-(26). It suffices to show that $(\phi, G)$ can be chosen such that among (20) and (21) at least one holds at equality for each $u<v$, and among (23) and (24) at least one holds with equality for each $u$. Such a solution is the output of the above dynamic program, and therefore, $G(1)$ is the optimal solution value, and the configuration is optimal. Notice that the conditions (i)-(v) ensure that the configuration is indeed a feasible solution for the lot-sizing problem.

It remains to show how to choose $(\phi, G)$. Let $(\phi, G)$ be arbitrary satisfying (i)-(v) and (19)-(26) with maximum $\sum_{u \neq v} \phi(u, v)+\sum_{v} G(v)$ value. Notice that this sum is finite, since (19)-(26) ensure that all the $\phi(u, v)$ and 
$G(v)$ values are bounded. Now suppose $\phi(u, v)<\min \left\{\left(\sum_{t=i_{j}}^{v-1} h_{t}^{2^{\prime}}\right) d_{v}^{2}+G(v+\right.$ 1), $\left.\left(\sum_{t=i_{j}}^{v-1} h_{t}^{2^{\prime}}\right) d_{v}^{2}+\phi(u, v+1)\right\}$ for some $u<v$. Obviously, $(u, v)$ is not among the values for which $\phi(u, v)$ is fixed by (i)-(v), since those values cannot be changed due to $(25)$. Notice also that $\phi(u, v)$ is involved only into two inequalities on the left hand side, i.e., there is one inequality in (20) and one in (21). Then we increase $\phi(u, v)$ until equality holds. Clearly, we obtain a solution with a larger sum of the values of $\phi$ and $G$, a contradiction. One similarly observes that none of the $G(v)$ values could be increased. Finally, if some of the $\phi(u, u)$ is strictly smaller than $f_{u}^{2}+\min \{G(u+1), \phi(u, u+1)\}$, then we may increase $\phi(u, u)$ along with all the $\phi(u, k)$ with $k<u$, to keep (22) satisfied, a contradiction again. Hence, this choice of $(\phi, G)$ is as desired. 